\title{
THE LURE OF INTERDISCIPLINARY
} ORTHODONTICS

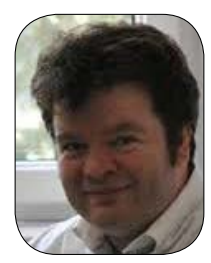

Alexandru Simion OGODESCU

DDS, PhD, Dr Habil

Professor \& Chairperson

Associate Editor Sections - Editor-in-Chief

Dear readers,

For 20 years I have been passionate about interdisciplinary collaboration between different specialties in dentistry and orthodontics. My doctoral studies, extensive research, publications, conferences, monographs have focused on interdisciplinary orthodontics and most of my daily clinical work as an orthodontist is dedicated to these interdiciplinary cases.

At first, few believed in this concept: my orthodontist colleagues were distrustful of the possibilities of adult orthodontics, periodontists did not believe that teeth with bone loss have an...,orthodontic future", prosthodontists were used to "straightening" teeth prosthodontically, while implantology was not a common therapeutic option. Often when I presented them with a case of interdisciplinary orthodontics, the attitude was rather more skeptical than enthusiastic.

The rescue came in 2003 at the Congress of the European Orthodontics Society (EOS) in Prague, when I heard Professor Vincent Kokich (USA) - one of the world's leading specialists in orthodontics, probably the most passionate supporter of interdisciplinary orthodontics and an excellent speaker in the field, insisting on how important it is, especially for the young generation of specialists, to "grow" together in interdisciplinary teams, where each member informs the other on the latest therapeutic possibilities in his specialty. This sincere and continuous mutual information has led to the elaboration of therapeutic principles and protocols of interdisciplinary collaboration through which each discipline assists the other, together offering higher quality medical services to the patient (Ogodescu A, 2006).

The internships attended at the Pedodontics-Orthodontics Department of the University of Zurich, Switzerland is where I learned from dear friends, Prof. Dr. Werner Frunz, Dr. Wanda Gnoinski, Dr. Rolland Maenchen, Prof. Dr. Marc Schaetzle how useful interdisciplinary collaboration can really be.

Almost no one at that time conceived of postponing prosthodontic treatment for 6-12 months to upright a lower second molar, to wait 3-6 months for the egression of a subgingivally decayed root, to correct a crossbite, a rotation, or to postpone insertion of an implant for 6 months to extrude a compromised root in order to create alveolar bone. In the case of patients with dento-maxillary abnomalies secondary to periodontal disease, no one saw orthodontics as a solution for their recovery either, but the fascination exercised by this new area of orthodontics was on its way and slowly it managed to convince more and more specialists.

As I stated in 2006 in my doctoral thesis, the implementation of this new concept is best summarized by the term TEAM ("together everyone achieves more") and the logo that I launched then for the new discipline is still valid today.

The success of establishing such a team of different specialties (orthodontics, periodontics, prosthodontics, maxillofacial surgery, occlusology, radiology, paedodontics) is based on a series of considerations: good communication, excellence in specialty, mutual respect and trust. I was lucky to have special colleagues in Timisoara with whom I could solve the most difficult cases. Together we trained, learned from each other and came to develop interdisciplinary cooperation protocols. In 2008 we published the first monograph on interdisciplinary orthodontic treatments in adults. For almost 10 years I have been teaching an optional course to sixth year students at the Faculty of Dentistry entitled: „Principles of interdisciplinary orthodontic treatment". 
Today I am really satisfied to watch my former students excel in the therapy of complex cases via an interdisciplinary approach. Young prosthodontics specialists no longer conceive of grinding sound teeth, instead they ask us, orthodontists, to recalibrate spaces for them to insert implants. The goals of preprosthodontic orthodontics have changed. Fellow periodontologists, with whom we work on the same substrate, the alveolar bone, have a completely different view on the advantages of orthodontic treatments in patients with a periodontal disease and the situations in which periodontal therapy favors orthodontic treatment. Implantologists have the patience to extrude compromised roots for implant site development, they help us to increase the anchorage in orthodontics, in the expansion of the jaw.

Patients have also learned the benefits of interdisciplinary orthodontics, many more adults are now asking for orthodontic treatments in combination with their periodontal or prosthodontic treatments.

Technology has evolved enormously, the digitalization of orthodontics is taking shape, and communication - so important in the interdisciplinary team - is easier today than ever.

Many dental clinics have specialist doctors who work together and share their knowledge to achieve better oral rehabilitation of patients.

My twenty-year old dream has come true. The appeal of interdisciplinary collaboration finally overcame the fear of communicating between colleagues from various specialties. The initial difficulties and distrust in the field of interdisciplinary orthodontics are a thing of the past and today excellence characterizes this dynamic field.

May this beautiful and complex specialty live a very long life as it has given me so much satisfaction throughout all these years!

Sincerely yours,

\author{
A.S. Ogodescu \\ DDS, PhD, Dr Habil, ORCIDiD: https://orcid.org/0000-0001-9797-7157 \\ Professor \& Chairperson \\ Senior specialist ODFO \\ Department of Pedodontics-Orthodontics \\ Faculty of Dental Medicine \\ "Victor Babeș" University of Medicine and Pharmacy Timișoara \\ Timișoara, Romania \\ Associate Editor Sections - Editor-in-Chief
}

